\title{
Computational Neuroscience
}

\author{
Oleg Yu Mayorov ${ }^{1,2,3^{*}}$ \\ ${ }^{1}$ Kharkiv Medical Academy of Postgraduate Education, Ministry of Health of the Ukraine, Ukraine \\ ${ }^{2}$ Institute for Medical Informatics and Telemedicine, Kharkiv, Ukraine \\ ${ }^{3}$ Research Institute for Children and Adolescents Health Protection of the National Academy of Medical Sciences, \\ Kharkiv, Ukraine
}

Correspondence to:

Professor Oleg Yu. Mayorov,

MD, PhD, Dr.Sci., Institute for Medical Informatics and Telemedicine, Kharkiv, Ukraine.

E-mail: o.y.mayorov@gmail.com
EJBI 2018; 14(1):2-3

Received: November 27, 2017

Accepted: December 12, 2017

published: January 10, 2018
One of the key components of computer neuroscience is the method of electroencephalography (EEG), which is widely used to study the activity of the human and animal's brain.

With the advent of the EEG, after a brief euphoria associated with the hope that this method will quickly reveal the secrets of the brain, an understanding emerged of the need to create and apply methods which can make a quantitative objective analysis of the electroencephalogram.

Quantitative EEG (qEEG) is an objective method of studying the central and peripheral nervous system, which is widely used in the clinic of internal diseases, neurology and psychiatry.

The qEEG significantly changed the classical EEG examination and gave qualitatively new possibilities for a quantitative assessment of the state of the nervous system.

This technology is developing very fast thanks to the achievements of microelectronics, computer technologies, communication technologies, mathematical methods of analysis and clinical informatics.

The effective use of encephalography for brain research and diagnosis, in the first place, depends on the qualitative recording of EEG signals. This is due to the understanding of the operation of hardware and software for recording EEG, compliance with the conditions in which the record is made in accordance with international standards. If these conditions are not met, the application of complex, expensive methods of mathematical analysis will not give the expected result. At the stage of EEG analysis it is necessary to choose one or several methods of analysis correctly depending on the task.

This requires a deep knowledge of the mathematical essence of the methods of analysis and capabilities available to the researcher of information diagnostic technologies for EEG analysis.

The most difficult stage is the neurophysiological and clinical interpretation, obtained as a result of the analysis of secondary mathematical indicators. This requires a deep knowledge of neuroanatomy, neurophysiology, and projection (extrapolation) of this knowledge with the neurological and psychiatric clinic.

One of the most sought-after areas is the algorithms of online and off-line detection of epileptiform activity for outpatient and 24-hour monitoring of patients. In addition, the development of a complex mathematical method of dipolar localization or the „solution of the inverse problem" makes it possible to establish a focus of epileptiform activity that is localized in the depth of the brain in subcortical structures, especially in the structures of the limbic system, which in some cases trigger epileptic seizures. There is a widespread introduction of EEG video monitoring to detect transient epileptiform activity and other transient neurological disorders. Video monitoring of EEG is also used to evaluate data for surgical treatment of seizures, and how intraoperative and resuscitative monitoring is in close contact with anesthesiology and neurosurgery.

Effective is the use of qEEG in sleep disorders for the recognition of sleep patterns - polysomnography, which is used to correctly diagnose and target treatment.

qEEG is used in newborns pathologies, pathological development of children and adolescents. The methods of qEEG are also used in the diagnosis of various clinical disorders: metabolic imbalance, endocrine disorders, toxic effects, coma, specific effects of drugs, and detection of brain death. The qEEG is used in conjunction with other methods of neuroimaging.

Neuropathologists and psychiatrists have a special need for methods of effective quantitative estimation of EEG for diagnosis and evaluation of the effectiveness of treatment of functional disorders - neuroses, psychoses, schizophrenia, when methods of medical visualization (tomography) are not effective.

As the power of personal computers increased, it became possible to implement complex mathematical algorithms and create new diagnostic technologies on their basis.

Widely used in the analysis of EEG methods of correlation, spectral and pair coherent analysis are not suitable for the study of cognitive functions of the human and animal brain. These functions are violated in children and require control over treatment and recovery - trauma, genetic disorders and congenital malformations.

There is an increase in the number of depressive and anxietyphobic disorders, including children and, especially, adolescents. To study the objective quantitative indicators of cognitive impairment, 
high efficiency is provided by the use of technologies based on multidimensional linear spectral analysis of the EEG.

However, the multidimensional linear analysis is the method for the analysis of linear systems and also the EEG considers as stochastic process. So, that does not allow evaluating comprehensively all EEG properties. Alternative to traditional approach is the hypothesis, according to which an EEG signal is necessary to consider as an output of non-linear system that is in a state of dynamic chaos.

For diagnostics of a condition of the brain or various mental diseases outcomes of the nonlinear analysis are extremely important. They - are objective and give the additional information. The basic information consists in evaluation of some "norm of chaos" ("norm of chaotic state"), and also in evaluation of deviations from "norm" (in this or that leg) under influence of those or other actions. It may be various mental, endocrine, neurological diseases, emotional stresses, which call deviations of a degree of a chaos (chaotic state) from "norm". Thus deviations in those and other legs may mean "illness".
If we will manage to reveal for given neurodynamic system (or an aspect of cerebral activity, for example, perception, memory, emotions, motivations etc.) for this or that cerebral process "norm" of a chaos deviations in both directions can be surveyed as "illness" and, hence, as a degradation. Further it is possible to inspect a choice of a technique of "treatment". Here the criterions of relative degree of chaotic state again enter in game. If by this criterion "treatment" approximates a condition of an open system to "norm", it means that process of self-organizing takes place. Otherwise "treatment" calls the further degradation. To solve the tasks described above the theoretical approach worked out, calculations algorithms, new secondary EEG (ESCoG) indices and their physiological interpretation are proposed to use.

The need to use sophisticated mathematical methods for EEG analysis for experimental and clinical studies today comes to the fore problems of standardization of methods, equipment and ongoing training of specialists - clinical neurophysiologists and technical personnel. 